\section{Why Are Physicists Involved in the Studies on the Origin of SARS-CoV-2?}

\author{
Ali Reza Mehdizadeh ${ }^{1,2}$, Joseph J Bevelacqua3, James S Welsh',5, Seyed Ali \\ Reza Mortazavi ${ }^{6}$, Leila Haghshenas7 , Seyed Mohammad Javad Mortazavi*i]
}

$\mathrm{P}$ hysics is a science that deals with the structure of matter and how the fundamental constituents of the universe interact. Physicists are scientists who study the nature, in particular, how matter and energy interact, and explore the physical laws controlling the world. Physicists apply their knowledge of science and mathematics to promote our understanding of the way the universe works, and in some cases they develop new technologies. During the COVID-19 pandemic, many physicists around the globe, used their expertise to control the disease by developing cutting-edge technologies such as those used in non-contact thermometers [1]. As discussed in the April 2020 issue of the "Physics World", these physicists are looking at how physics may play a crucial role in the fight against the pandemic [2]. Moreover, in the June 2020 issue of Physics World, James McKenzie explained how the lockdown showed us that our communication technology operates very well [3]. Due to profound impact of COVID-19 on our community, in some countries the physics societies have decided to fund projects that address critical needs resulting from the COVID-19 pandemic [4]. Furthermore, researchers have reported that the interactions between B cells and COVID-19 can provide an ideal working model for statistical physics as well as modern biology [5]. Although statistical physics basically focuses on the behavior of large collections of interacting objects (e.g. atoms and molecules), it has recently expanded into new areas such as biology [5].

As of June 9, 2021, SARS-CoV-2, the RNA coronavirus that caused the pandemic, has affected over 174 million people globally, with more than 3.75 million reported deaths.

A recent report published in the Wall Street Journal states that SARS-CoV-2 has a genetic footprint that has never been found in other coronaviruses with a natural origin. This report notes that evidence supporting the concept that the coronavirus may have escaped from a virology lab is now more credible compared to the early unfounded rumors and hearsay.

Dr. Steven Quay and Professor Richard Muller, a physics professor at the University of California Berkeley, in their Opinion/Commentary article, noted two key pieces of evidence to support their claim of SARS-CoV-2 being manufactured in a laboratory. As the most compelling reason to support their lab leak hypothesis, they state that SARS-CoV-2 has the genome sequencing 'CGC-CGG' or the socalled 'double CGG' which has never been naturally found in the whole class of coronaviruses up to this point.

It is worth noting that codon preferences are different in different organisms. While in human cells CGT, CGC or CGG are common codons for designating arginine, CGG is the least popular codon for arginine in coronaviruses and the 'double CGG' has never found in any other beta-coronavirus [6].

Although we know that recombination serves as a common method of acquiring new capabilities in different viruses, it is evident that SARS-CoV-2 could not have picked up a genome sequence from other viruses if that sequence did not already exist in any other virus. While the swap hypothesis (recombination) is ruled out, it
${ }^{1} \mathrm{MD}$, PhD, Editor-in-Chief of

the Journal of Biomedical

Physics and Engineering,

Shiraz University of Medical

Sciences, Shiraz, Iran

${ }^{2} \mathrm{MD}, \mathrm{PhD}$, lonizing and

Non-lonizing Radiation

Protection Research Center

(INIRPRC), Shiraz Univer-

sity of Medical Sciences,

Shiraz, Iran

${ }^{3} \mathrm{PhD}$, Bevelacqua Resourc-

es, Richland, Washington

99352, United States

${ }^{4} \mathrm{MD}, \mathrm{PhD}$, Department of

Radiation Oncology Edward

Hines Jr VA Hospital Hines,

Illinois, United States

${ }^{5} \mathrm{MD}$, PhD, Department of

Radiation Oncology, Stritch

School of Medicine, Loyola

University, Chicago, IL,

United States

${ }^{6} \mathrm{MD}$, School of Medicine,

Shiraz University of Medical

Sciences, Shiraz, Iran

${ }^{7} \mathrm{PhD}$, Postdoc association

member of Harvard Medi-

cal School, Boston, MA,

United States

${ }^{8} \mathrm{PhD}$, Department of Medi-

cal Physics and Engineer-

ing, School of Medicine,

Shiraz University of Medica

Sciences, Shiraz, Iran

*Corresponding author:

Seyed Mohammad

Javad Mortazavi

Department of Medical

Physics and Engineering,

School of Medicine, Shi-

raz University of Medical

Sciences, Shiraz, Iran

E-mail: mortazavismj@

gmail.com

Received: 26 June 2021

Accepted: 3 July 2021 


\section{Ali Reza Mehdizadeh, et al}

might still be plausible that the 'double CGG' genome sequence has been acquired at random, through adaptive mutations. However, this combination is rare in nature. In contrast, the human genome CGG sequence is the common arginine codon. Given this consideration, the laboratory escape theory receives more support.

A recent Nature report, which heated up the lab leak debate "The COVID lab-leak hypothesis: what scientists do and don't know", states that besides the US, Australia, the European Union, and Japan have also called for a robust investigation into the origins of SARS-CoV-2. The influence of political considerations in the debate is not helpful, and the origin discussion should be objectively and thoroughly evaluated and openly monitored without preconceived ideas or political biases. Interestingly, the Nature report has no specific conclusion at the end and asks the readers to wait weeks, months, and years to complete the evidence and clarify the issue. However, in this report, the CGG sequence issue is alluded to as a "smoking gun"! It should be noted that for the furin cleavage site to have appeared spontaneously in SARS-CoV-2, a long and complicated chain of events must have happened and that each individual event was improbable. In contrast, the 'double CGG' codon might not be unexpected in a lab escape scenario [6, 7].

Moreover, if we accept that spontaneous mutation was involved in the 'double CGG' genome sequence, the role of selective pressure in viral evolution, as discussed as early as March 2020 by our team of physicists receives more support [8]. When our team of physicists and medical doctors proposed low dose radiation therapy (LDRT) as an absolutely 'selective pressure-free' treatment method for COVID-19 associated pneumonia [9], we raised red flags about treatment methods that could lead viruses such as SARS-CoV-2 to evolve through 'selective pressure' mediated mutations $[10,11]$.

We note in closing that no definitive conclusions regarding the origins of COVID-19 are possible without a full disclosure of all relevant data. Given the worldwide impact of the virus, full data disclosure is a scientific imperative. These data require an unbiased evaluation without preconceived notions. At this juncture, the discovery of the 'double CGG' sequence is consistent with unnatural origin, and must be thoroughly evaluated if we are to ever determine the definitive source of COVID-19. Given the available data, a laboratory escape scenario cannot be excluded.

\section{Conflict of Interest}

None

\section{References}

1. IOP. Stories from physicists helping to tackle COVID-19. IOP; 2021. Available from: https://www.iop.org/stories-physicists-helping-tackle-covid19\#gref.

2. Physics World. COVID-19: how physics is helping the fight against the pandemic. Physics World; 2020. Available from: https://physicsworld.com/a/ covid-19-how-physics-is-helping-the-fight-against-the-pandemic/.

3. McKenzie J. Why COVID-19 could make physicists kick some of their habits forever. Physics World; 2020. Available from: https://physicsworld.com/a/ why-covid-19-could-make-physicists-kick-some-of-their-habits-forever/.

4. APS. APS's Efforts to Help Physics Community Recover from COVID-19 Impact. The American Physical Society (APS); 2021. Available from: https:// www.aps.org/about/covid-19/.

5. Lobos I. The PHYSICS OF A DEADLY VIRUS, Immune response research hints at answers to fighting deadly coronavirus. University of Washington; 2021. Available from: https://www.washington.edu/uwit/stories/immune-response-research-coronavirus/.

6. Wade N. The origin of COVID: Did people or nature open Pandora's box at Wuhan? Bulletin of the Atomic Scientists; 2021.

7. Kaina B. On the Origin of SARS-CoV-2: Did Cell Culture Experiments Lead to Increased Virulence of the Progenitor Virus for Humans? In Vivo. 2021;35(3):1313-1326. doi: 10.21873/inviv0.12384. PubMed PMID: 33910809. PubMed PMCID: PMC8193286.

8. Ghadimi-Moghadam A, Haghani M, Bevelacqua JJ, Jafarzadeh A, et al. COVID-19 tragic pandemic: concerns over unintentional "directed accelerated evolution" of novel Coronavirus (SARS-CoV-2) and introducing a modified treatment method for ARDS. J Biomed Phys Eng. 2020;10(2):241-6. doi: 10.31661/jbpe.v0i0.2003-1085. PubMed PMID: 32337192. PubMed PMCID: PMC7166223.

9. GBevelacqua JJ, Mehdizadeh AR, Mortazavi SAR, Mortazavi SMJ. A New Look at the LDRT treatment for COVID-19 Associated Pneumonia: The Issues of Antiviral Resistance and Virus Spread-Ability. J Biomed Phys Eng. 2020;10(5):549-52. doi: 10.31661/jbpe.v0i0.2007-1151. PubMed PMID: 33134212. PubMed PMCID: PMC7557455.

10. Mortazavi SAR, Mortazavi SMJ, Sihver L. Selective Pressure-Free Treatments for COVID-19. Radiation. 2021;1(1):18-32. doi: 10.3390/radiation1010003.

11. Mortazavi SMJ, Kefayat A, Cai J. Low-dose radiation as a treatment for COVID-19 pneumonia: A threat or real opportunity? Medical Physics. 2020;47(9):3773-6. doi: 10.1002/mp.14367.

Citation: Mehdizadeh AR, Bevelacqua JJ, Welsh JS, Mortazavi SAR, Haghshenas L, Mortazavi SMJ. Why Are Physicists Involved in the Studies on the Origin of SARS-CoV-2? J Biomed Phys Eng. 2021;11(4):413-414. doi: 10.31661/jbpe.v0i0.2106-1361. 